\title{
Entropy-regularized deconvolution of cellular cryotransmission electron tomograms
}

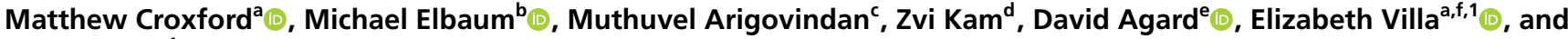 \\ John Sedat ${ }^{\mathrm{e}, 1}$ \\ ${ }^{a}$ Section of Molecular Biology, Division of Biological Sciences, University of California San Diego, La Jolla, CA 92093; ${ }^{b}$ Department of Chemical and \\ Biological Physics, Weizmann Institute of Science, Rehovot 760001, Israel; ' Department of Electrical Engineering, Indian Institute of Science, Bengaluru \\ 560012 , India; ${ }^{d}$ Department of Molecular Cell Biology, Weizmann Institute of Science, Rehovot 760001, Israel; ${ }^{2}$ Department of Biochemistry and Biophysics, \\ University of California, San Francisco, CA 94158; and ${ }^{f} \mathrm{HHMI}$, University of California San Diego, La Jolla, CA 92093
}

Contributed by John Sedat, October 21, 2021 (sent for review May 10, 2021; reviewed by Wah Chiu and Nikolaus Grigorieff)

Cryo-electron tomography (cryo-ET) allows for the high-resolution visualization of biological macromolecules. However, the technique is limited by a low signal-to-noise ratio (SNR) and variance in contrast at different frequencies, as well as reduced $Z$ resolution. Here, we applied entropy-regularized deconvolution (ER-DC) to cryo-ET data generated from transmission electron microscopy (TEM) and reconstructed using weighted back projection (WBP). We applied deconvolution to several in situ cryo-ET datasets and assessed the results by Fourier analysis and subtomogram analysis (STA).

cryo-electron tomography | deconvolution | subtomogram analysis | structural biology | missing wedge

$\mathbf{R}$ ecent advances in cryo-electron tomography (cryo-ET), most notably the ability to thin cryopreserved specimens using a focused ion beam (FIB), have opened windows for the direct visualization of the cell interior at nanometer-scale resolution (1-9). Cells are rapidly frozen to achieve a vitreous form of ice that preserves biological molecules in a near-native state. They are then cryo-FIB milled to a suitable thickness of 100 to 350 $\mathrm{nm}$ for imaging with transmission electron microscopy (TEM). A series of projection images is acquired, typically with $1^{\circ}$ to $5^{\circ}$ increments, and then reconstructed into a three-dimensional (3D) volume (10). This 3D reconstruction is rendered for display and analysis, which may entail segmentation to highlight extended structures or averaging of subvolumes for enhancement of molecular-scale resolution (11-13).

While cryo-ET offers unparalleled resolution of cellular interiors, it is challenging for a number of reasons. First, vitrified biological samples are highly sensitive to damage by the electron irradiation required for imaging. Constraints on the permissible exposure result in limited contrast and a low signal-to-noise ratio (14). Additionally, higher-resolution information is degraded by radiation damage over the course of imaging (15), although approaches such as dose-symmetric acquisition have been developed to optimize recording of high frequencies (16). Second, the modality of wide-field TEM depends on defocus to generate useful phase contrast, but with a nontrivial dependence on spatial frequency that is expressed in a contrast transfer function (CTF). Contrast is lost at low spatial frequencies and oscillates at high spatial frequencies, meaning that material density could be represented as intensity either darker or lighter than background (17-19). Postprocessing is applied to correct this representation in the image intensities. The correction is inherently approximate and is especially challenging in tomography, where the defocus varies across the field of view for tilted specimens (20). Third, the available raw data are never sufficient to produce an unambiguous reconstruction. The tilt range is restricted by the slab geometry, typically to about $120^{\circ}$ around the vertical. The projected thickness of a slab also increases with tilt angle, resulting in degraded contrast and resolution from these contributions to the reconstruction. The missing information is best recognized in the kxkz plane of Fourier space, corresponding the $\mathrm{XZ}$ plane in real space, where it is known as the missing wedge. The gaps between discrete tilt angles also leave small missing wedges as seen in Fig. 1. Since the reconstruction is equivalent to an inversion in Fourier space, it is obvious that some interpolation is required and that the data are incomplete. As such, it is not surprising that different algorithms can generate somewhat different reconstructions from the same data. Commonly recognized artifacts are elongation along the $\mathrm{Z}$ direction and streaks projecting from high contrast points into neighboring planes in the volume.

In addition to the missing wedges, TEM images require a significant defocus to generate adequate contrast, and the process of reconstruction by weighted back projection (WBP) introduces well-known problems. These include significant intensity above and below the sample volume, where we expect vacuum with no signal. This is due to cross-terms in the WBP coming from the tilt wedges, as well as distortions in the WBP arising from the missing wedge. Because of these issues with cryo-ET data, filters to improve contrast and compensate for the missing wedge are an area of ongoing research (21). These techniques include nonlinear anisotropic diffusion, convolutional neural networks based on detector noise models, wavelet-based filtering methods, different implementations of deconvolution, and model-based iterative reconstruction (22-31). Here, we present a deconvolution approach to achieve both enhanced signal-to-noise ratio and missing wedge compensation.

The image distortions resulting from the incomplete tilt series and CTF can be characterized in terms of a single sample point in the data. This model is referred to as the point spread function (PSF), of which the hourglass PSF in light microscopy is a classic

\section{Significance}

Cellular cryo-electron tomography suffers from severely compromised $Z$ resolution due to the missing wedges of information not collected during the acquisition of tilt series. This paper shows that application of entropy-regularized deconvolution to transmission electron tomography substantially fills in this missing information, allowing for improved $\mathrm{Z}$ resolution and better interpretation of cellular structures.

Author contributions: M.C., M.E., E.V., and J.S. designed research; M.C. and J.S. performed research; M.C., M.E., M.A., Z.K., D.A., E.V., and J.S. analyzed data; and M.C., M.A., Z.K., D.A., E.V., and J.S. wrote the paper.

Reviewers: W.C., Stanford University; and N.G., University of Massachusetts Medical School.

The authors declare no competing interest.

This open access article is distributed under Creative Commons AttributionNonCommercial-NoDerivatives License 4.0 (CC BY-NC-ND).

${ }^{1}$ To whom correspondence may be addressed. Email: evilla@ucsd.edu or sedat@msg.ucsf.edu.

This article contains supporting information online at https://www.pnas.org/lookup/ suppl/doi:10.1073/pnas.2108738118/-/DCSupplemental.

Published December 7, 2021. 


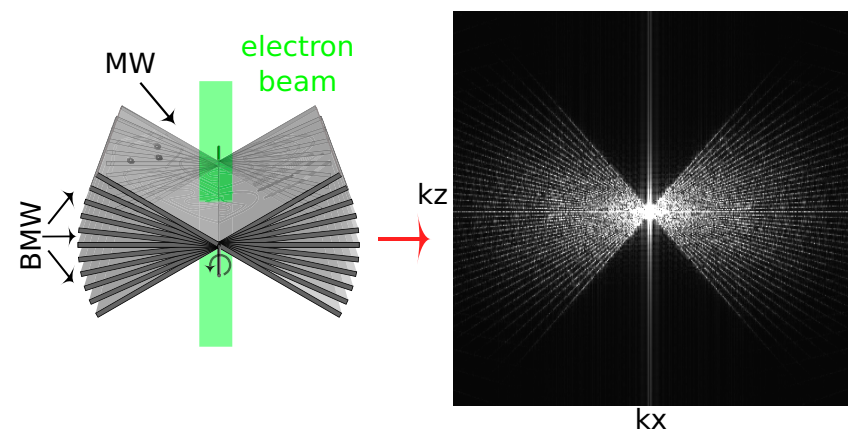

Fig. 1. Tilt series collection and the missing wedge issue. (Left) Schematic of the tilt series collection scheme. Sample projections are acquired over a range of tilt angles, typically from $-60^{\circ}$ to $+60^{\circ}$. (Right) The middle slice of the kxkz plane shows the missing wedge (MW) and baby missing wedges (BMWs) of information visualized in Fourier space. Attenuation of high frequencies due to radiation damage as the tilt series progresses is not depicted $(15,16)$.

example (32-34). Formally, the PSF is convolved with all points in the specimen function to form what is recorded in the image (35). If the PSF is well defined, it becomes possible to partially reverse the process of convolution to obtain an improved reconstruction. This reversal is referred to as deconvolution, which is a mathematical/computational iterative inversion processing procedure, extensively utilized in astronomy, spectroscopy, and light microscopy to partially restore data distorted by the imaging process (35). The deconvolution process is constrained. The most common constraint is the imposition of positivity of the deconvolved data (35). Other stabilizing constraints may include smoothing in real space to suppress high-frequency oscillations. Deconvolution is also very sensitive to noise, and most deconvolution algorithms include regularization parameters whose values are difficult to evaluate theoretically. Additionally, in most cases the deconvolution algorithms will diverge with increasing iterations, building up mottle and noise that obscure the interpretation of the final deconvolved image. Finally, most deconvolution implementations do not have a practical estimate of the error in the converged solution.

Entropy-regularized deconvolution (ER-DC) (36) is formulated to handle data with a weak signal-to-noise ratio, with a regularization term that exploits certain characteristics specific to images originating from crowded molecular environments such as cells. Specifically, in cellular images, high intensities and high second-order derivatives exhibit certain sparse distribution, and this property is exploited by the custom regularization used in ER-DC (36). This regularization was originally designed for fluorescence images, and this approach was taken recently for processing of scanning transmission electron microscopy (STEM) cryo-tomography reconstructions (29).

Since TEM is currently the dominant modality for biological 3D imaging of cells (37), it deserves a separate study, which is the focus of this paper. The major distinction is that the contrast inversions, which were absent in the STEM data as acquired for tomography, should be accommodated in the construction of the 3D PSF for TEM tomography. Here, a similar approach is taken for TEM. The combined effect of the two-dimensional (2D) CTF of the system and the missing wedge is captured in the form of 3D PSF. We attempt to remove the distortions caused by the 2D CTF and the missing wedge by deconvolving the back-projected 3D image with the aforementioned 3D PSF. We apply this approach to both real data, shown below, as well as idealized simulated data, discussed in SI Appendix (SI Appendix, Figs. S6 and S7) and examine the effects of ER-DC on real space qualitatively, by Fourier analysis, and by subtomogram averaging.

\section{Results}

Electron Tomography PSF. The key to a meaningful deconvolution is that the synthetic PSF should represent as closely as possible the 3D image of an ideal point source. In the case of TEM, this requires an accounting for the defocus imposed in the image acquisition, which is customarily expressed in terms of a CTF. The 3D PSF for deconvolution was computed from simulated projections of a point source with the same dimensions and pixel spacing as the aligned tilt series (Fig. $2 A$ ). The CTF was first convolved with a projected point source (Fig. $2 B$ ), and then, a synthetic tilt series was reconstructed to the same dimensions as the original tomogram using the tilt angles represented in the corresponding reconstruction (Fig. $2 C$ ). This is the real-space PSF, whose 3D fast Fourier transform (FFT) serves as the optical transfer function, or kernel, for the deconvolution (Fig. 2D). The 2D CTFs vary with the gradient of defocus for each micrograph in the tilt series.

Tomogram Deconvolution. As a first demonstration of cryo-ET deconvolution, we used a human embryonic kidney (HEK) cell cultured on grid that had been FIB milled to 150-nm thickness. The reconstructed volume contains membranes, microtubules, and a prominent crystalline protein array. The cells were overexpressing human Parkinson's-related protein LRRK2-I2020T (38), and the observed repetitive structure is likely an autophagosome, given its double-lipid bilayer structure (39). Contrast is sharp in slices through the XY plane of the tomogram, as expected (blue plane midstructure in Fig. $3 B$ ), but contrast and resolution in the $\mathrm{Z}$ direction, seen in a slice through the $\mathrm{XZ}$ plane (orthogonal green plane in midstructure in Fig. 3C), are severely compromised. Furthermore, the reconstructed volume displays a signal both above and below the specimen when observed in the XZ plane. Since the milled slab of material is finite in the $\mathrm{Z}$ direction and the sample is imaged in a vacuum, there should be negligible intensity outside the sample volume in the reconstructed data. This is a known artifact of WBP. These image distortions in real space can also be characterized in Fourier space, where the real-space dimensions $(x, y, z)$ correspond to the Fourier dimensions $\left(K_{x}, K_{y}, K_{z}\right)$. The protein array in the real-space XY plane appears as a lattice of calculated diffraction spots in the plane $\left(K_{x}, K_{y}\right)$, as expected (Fig. $3 E$ ). In the XZ plane, the lattice of spots is sharply truncated at the Fourier planes normal to the limits of the acquired tilts. In summary, WBP suffers from major distortions visible in both real and Fourier space.

The result of $3 \mathrm{D}$ deconvolution is shown alongside the reconstruction in Fig. 3. Full details appear in SI Appendix. All processing was performed using the PRIISM image processing software (40). Briefly, the ER-DC algorithm from PRIISM was
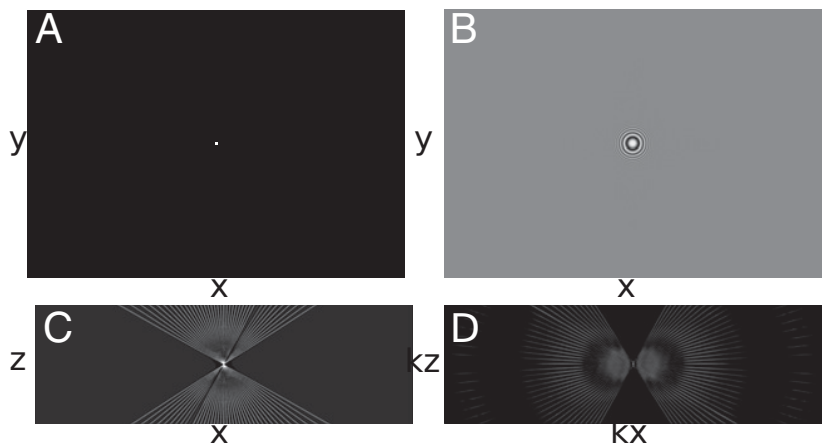

Fig. 2. Generating the TEM PSF. (A) Synthetic tilt series of a centered point source. $(B)$ Point source tilt series convolved with CTF. (C) Slice of the weighted WBP tomogram of the convolved CTF point source (PSF; $x z$ view). (D) A 3D FFT of the tomogram shown in C (xz slice). 
B
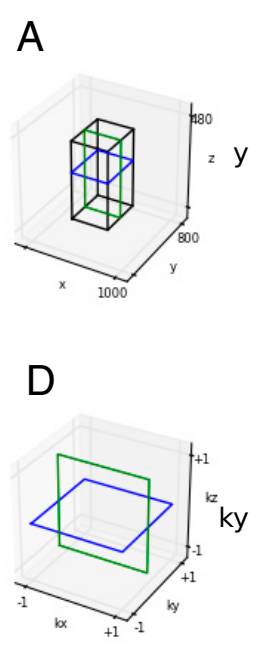

E
C

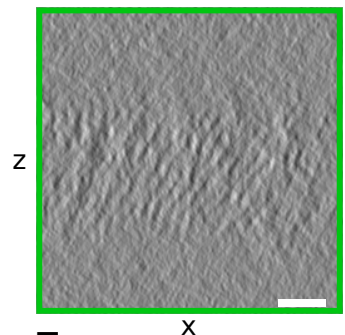

$\mathrm{F}$

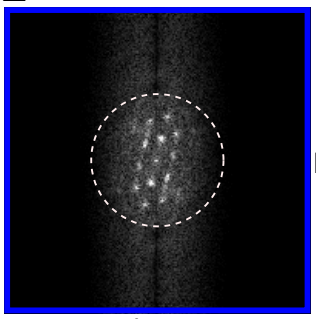

G

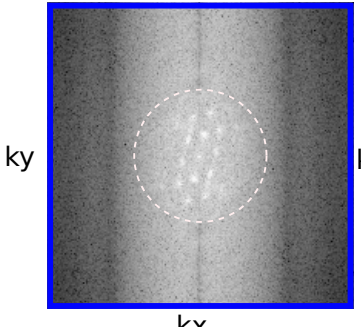

kx

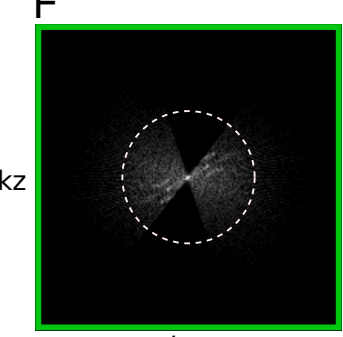

$\mathrm{kx}$

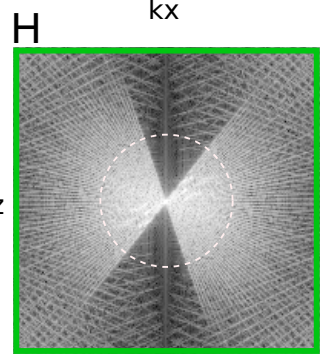

$\mathrm{kX}$
I

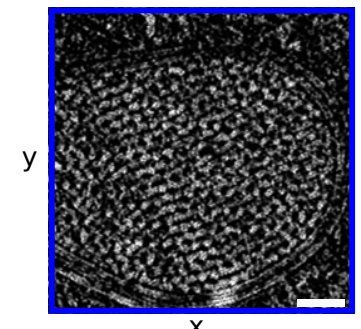

K

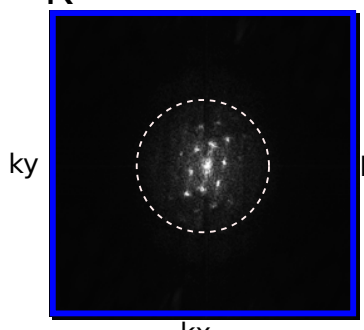

M

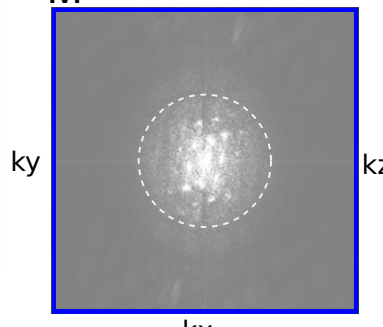

kx
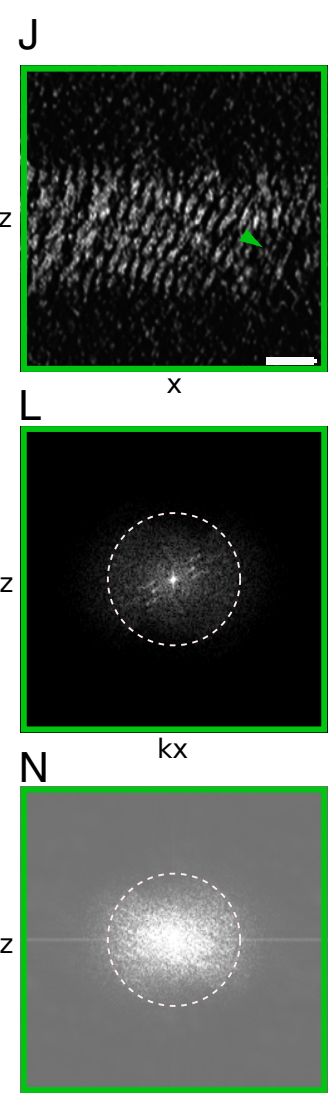

kx

Fig. 3. Filling of the missing wedge by deconvolution. (A) A schematic of the slices used to generate $B, C, I$, and $J$. (B) $X Y$ slice of a tomogram of an HEK cell reconstructed using WBP. Throughout this work, white intensities correspond to high-density values. (C) XZ slice of the same tomogram. (D) The schematic shows the slices through Fourier space used to generate $E, G, K$, and $M$ in blue and $F, H, L$, and $N$ in green. ( $E$ and $G$ ) Central slice of the $3 D$ FFT of the WBP. kxky plane shown at two different distributions of voxel intensities. ( $F$ and $H$ ) Central slice of the 3D FFT of the deconvolution. kxkz plane shown at two different distributions of voxel intensities. $(I-N)$ Corresponding results for the tomogram after deconvolution. Green arrow indicates the double lipid bilayer now visible. For reference, dashed circles indicate $0.03-\AA^{-1}$ resolution. (Scale bars: $100 \mathrm{~nm}$.)

applied using the simulated PSF. While contrast is enhanced in the $\mathrm{XY}$ plane, the more striking improvement is seen in the $\mathrm{XZ}$ plane (Fig. $3 J$ ) in comparison with the WBP (Fig. 3C). In the deconvolved tomogram, two lipid bilayers are visible (Fig. $3 J$, arrow) across the entire sample along $\mathrm{Z}$, as is the crystalline array (Fig. 3J). The restoration of information along $\mathrm{Z}$ in real space can also be seen in the 3D Fourier transform of the deconvolved volume, which shows increased signal in the previously empty regions corresponding to the missing wedges (Fig. $3 F$ and $L$ ).

A very effective way to observe the results of deconvolution is to study a small volume of the WBP and/or deconvolved in a dynamic interacting display module, typically a video of the rotating volume. Stereo pairs with additional rotated views are shown for the WBP and deconvolution (Movies S1 and S2). These may be rocked with a cursor bar, as described in SI Appendix, in order to gain an impression in 3D. Distortions along the $z$ axis associated with the WBP are largely removed after deconvolution. Significant information in the power spectrum appears beyond a spatial frequency of $\sim 2.5 \mathrm{~nm}$, which corresponds nominally to the second zero in the CTF for a $6-\mu \mathrm{m}$ defocus.

A Second Deconvolution Example. For a second example, we applied ER-DC to a tomogram of a relatively thick lamella of Saccharomyces cerevisiae cells $(370 \mathrm{~nm})$. Other than the thickness, cryo-ET data of nuclei are challenging samples to interpret as nuclei are densely packed and lack high-contrast features like membranes, cytoskeletal filaments, or large and defined particles such as ribosomes. As with the deconvolution of mammalian cells, deconvolution provided increased contrast in $\mathrm{XY}$ and an improved ability to visually interpret information along $\mathrm{Z}$ compared with the WBP. The nuclear envelope is clearly visible in the XY slices of the WBP and the two deconvolutions (Fig. 4 $A, E$, and $I)$. In XZ, however, no clear structure can be followed in the BP (Fig. 4C), but it can be more easily followed in the deconvolution (Fig. $4 G$ and $K$ ). Additionally, the missing wedge seen in Fourier space is filled in by the deconvolution process (Fig. $4 \mathrm{H}$ and $L$ ). By utilizing rotating angle stereopair renderings of the volume, one can compare the WBP and deconvolved volumes in 3D (Movies S3 and S4). In the BP, there is little distinguishable structure as the volume rotates. In contrast, fine features can be identified at every angle, such as the nuclear envelope, as well as densities that could correspond to chromatin and nucleosomes. The 3D FFT of the deconvolution (Fig. 4), kxkz view, shows the missing wedges being filled in, indicating that the deconvolution process helps correct for these artifacts, even in challenging samples.

Deconvolution and Subtomogram Analysis. Subtomogram analysis is an approach to protein structure determination in situ (11, 41-55). Similarly to single-particle analysis, of which it is an extension to $3 \mathrm{D}$, averaging multiple examples of images that represent particles of the same kind serves to reduce noise. If the molecules lie in random orientations, 3D averaging can also be used to compensate for the missing wedge (11). The crystallinelike body seen in Fig. 3 provided an interesting test case for averaging where orientations were determined to be uniform by 

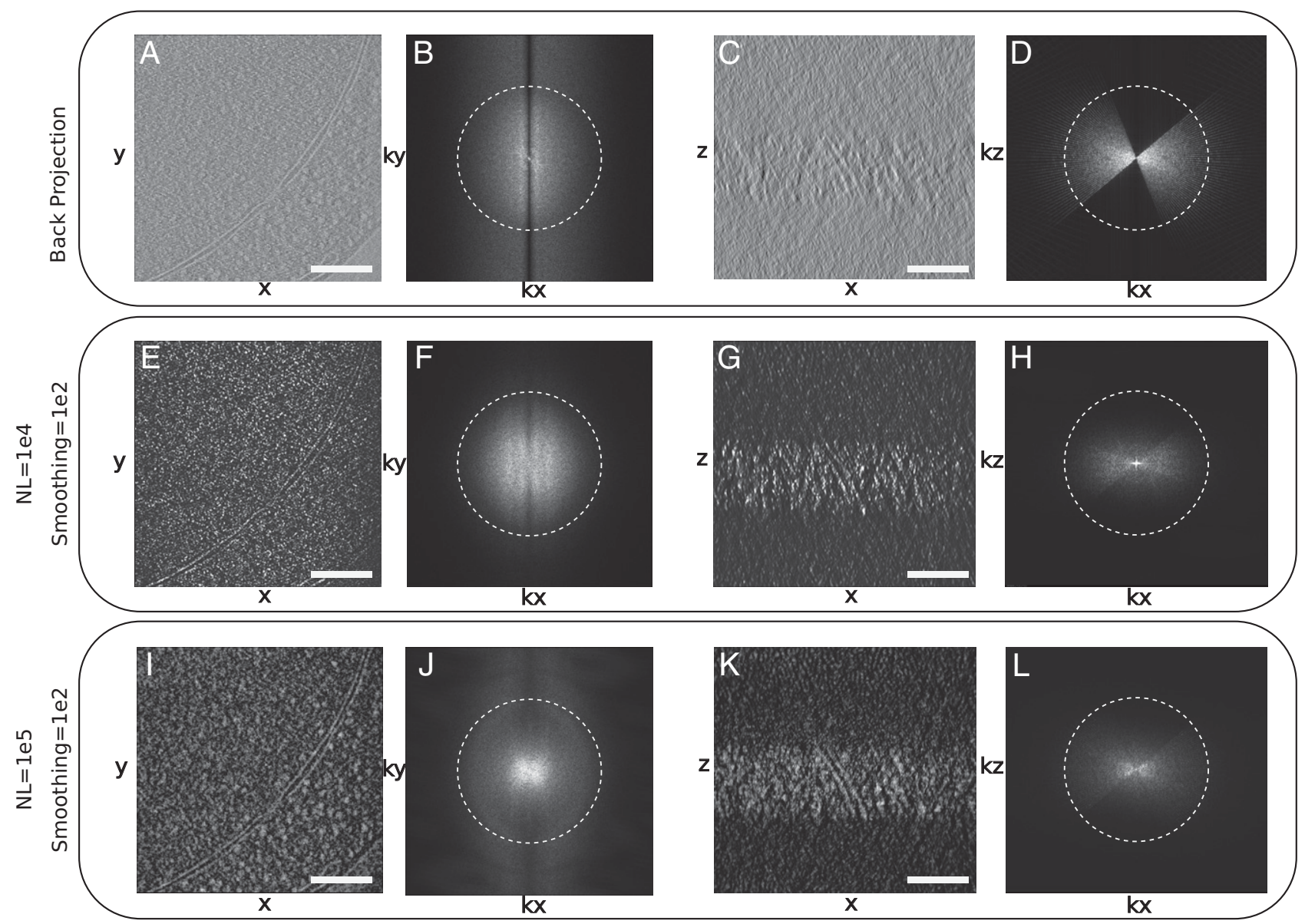

Fig. 4. Deconvolution of a tomogram of the nuclear periphery of an S. cerevisiae yeast cell. $(A)$ Central slice (10.6-nm thick) of the $X Y$ plane of a WBP tomogram. (B) Fourier transform of $A$. (C) Central slice of the XZ plane of the WBP tomogram. (D) Fourier transform of C. I Central slice of the XY plane of the tomogram deconvolved with a smoothing parameter of 100 and a nonlinearity factor of 10,000 . (F) Fourier transform of $E$. (G) Central slice of the $X Z$ plane of the deconvolved tomogram. (H) Fourier transform of $G$. (I) A 10.6-nm slice of the XY plane of the tomogram from A, deconvolved with a smoothing parameter of 100 and a nonlinearity factor of 100,000. (J) Fourier transform of $I$. (K) A 10.6-nm slice of the XZ plane of the deconvolved (DC) tomogram from I. (L) Fourier transform of $K$. Dashed circles indicate $0.03-\AA^{-1}$ resolution. (Scale bars: $100 \mathrm{~nm}$.)

translational symmetry in the crystal (Fig. 5). Therefore, only select orientations are represented in the sample. First, we attempted to align the crystal subunits over $360^{\circ}$ in $\theta$ and $\phi$ on the

WBP reconstruction. This resulted in an alignment that was dominated by the missing wedge, a common pitfall in subtomogram averaging, and produced a structure that was strongly elongated

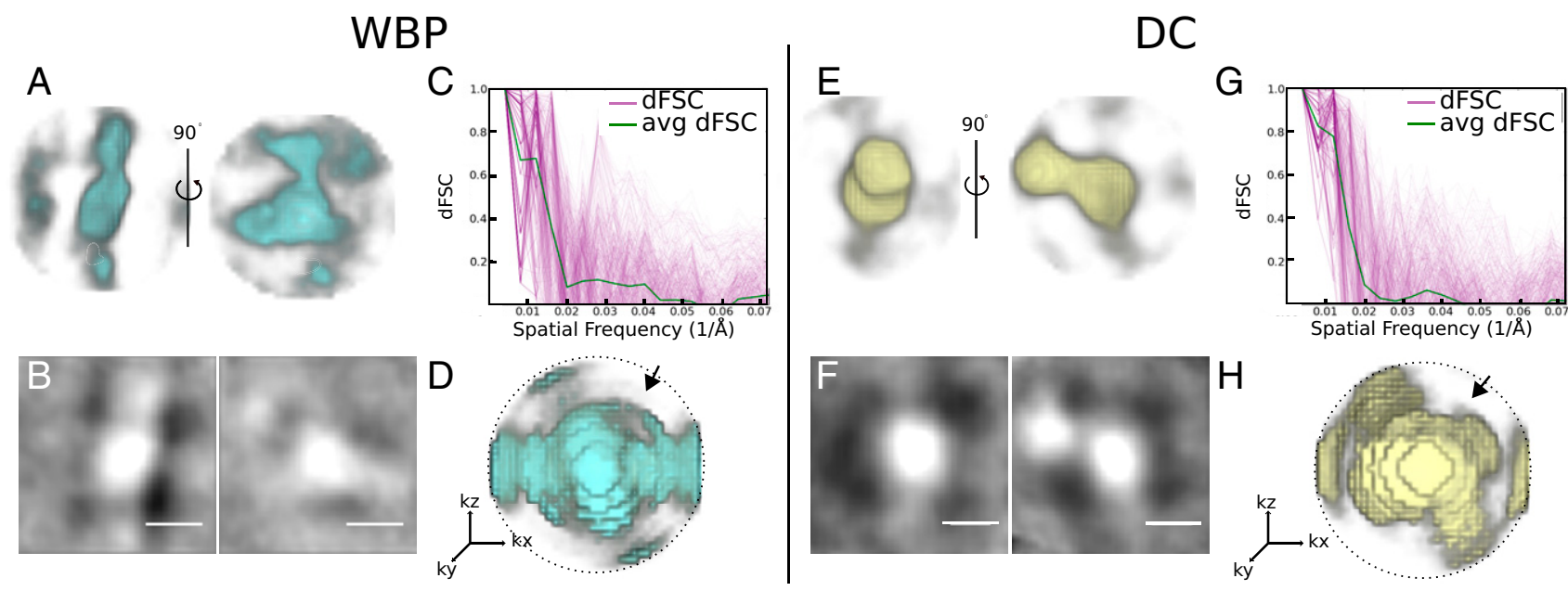

Fig. 5. Comparison of subtomogram analysis of a crystalline body in WBP vs. deconvolved reconstructions. (A) Two views of the crystal body average of WBP subtomograms. (B) The central 1.4-nm slice of the WBP crystal body subtomogram average. (C) The D FSC curves generated from two half-map averages of the WBP crystal body subtomogram average. The green line is the average, and pink lines are individual dFSCs. (D) A 3D render of dFSC curves; the arrow denotes the center of the missing wedge. $(E-H)$ Corresponding averages and FSCs derived from the deconvolved (DC) volume. dFSCS are shown out to Nyquist resolution $\left(0.07 \AA^{-1}\right)$. (Scale bars: $10 \mathrm{~nm}$.) 
(Fig. 5A). Second, we used the same particles but this time from the deconvolved dataset. Alignment and averaging resulted in a structure that resembled much better the unit of the crystalline array in the original tomogram (Fig. $5 E$ ). Third, we averaged the WBP particles using the transformations determined by the deconvolution alignment. In this last case, we obtained a structure similar to the one obtained from deconvolution-aligned and averaged particles (SI Appendix, Fig. S4B), demonstrating that the alignment of subtomograms is improved by deconvolution. This tomogram was acquired from an HEK cell overexpressing human LRRK2 (38). While the identity of the molecules forming the crystalline-like array was not specifically established (e.g., by correlative light and electron microscopy) and the number of particles in this tomogram is severely limited (82), the overall shape of the deconvolved average resembles the cryo-electron microscopy (cryo-EM) structures of LRRK2 determined both in situ bound to microtubules (38) and in vitro (56).

Fourier shell correlation (FSC) is widely used in single-particle cryo-EM (57) as a metric of the resolution of a molecular structure. It is a quantitative measure of similarity, typically implemented in cryo-EM by comparing two structures, each generated from a half dataset. The comparison is done by correlating shells of the 3D Fourier transform of each of the half maps. Standard FSC compares global similarities, correlating all orientations contained within a shell, giving a single curve for the entire structure as a function of spatial frequency. Resolution is then quoted as the inverse spatial frequency where the correlation drops below an accepted threshold. Directional Fourier shell correlation (dFSC) is a variant in which all Euler angles are explored for frequency comparison and provides a representation of resolution in all directions (58). dFSC was applied to two halfmap averages from the crystalline array in the WBP and then in the deconvolved averages to assess changes in resolution in any direction between the WBP (Fig. $5 C$ ) and deconvolution (Fig. 5G). The resolution from the averaged dFSC curves is similar for the deconvolved and the WBP reconstructions $(\sim 53 \AA)$ using the gold standard FSC; however, the curves of the averaged and individual dFSCs have higher correlation at this and higher resolutions. This is evident when comparing the $3 \mathrm{D}$ rendering of the dFSC for the WBP (Fig. 5D) and deconvolution (Fig. 5H), where the resolution is anisotropic (lower correlation in the area of the missing wedge denoted by the arrow in Fig. $5 H$ ) for the WBP but not for the deconvolution. Further, higher resolution is found in the deconvolution reconstruction.

To investigate the effects of deconvolution on the alignment of the particles and the improvement of the average due to the missing wedge separately, we chose to use microtubules since their structure is well established, as are the pipelines for subtomogram analysis. We analyzed a tomogram of reconstituted microtubules decorated by the Parkinson's-related protein LRRK2 ${ }^{\mathrm{RCKW}}$ (56). In the tomogram, it is evident that the deconvolution process increased the contrast between the microtubules and the surrounding media, and we again see a reduction in XZ distortions (Fig. $6 A, B, D$, and $E$ ), as well as a corresponding filling of information in the missing wedge in Fourier space (Fig. $6 C$ and $F$ ). Microtubule subtomograms were extracted from both the WBP and deconvolved volumes using the filament tracing function in Dynamo (59). The subtomograms were independently aligned and averaged as described in ref. 38 and in Materials and Methods (Fig. $7 A$ and $B$ ). Note that the contrast between protofilaments is distinctly sharper for the deconvolved data. However, this method of alignment includes an azimuthal randomization that is specifically designed to average out the missing wedge in the final average. To assess the effect of deconvolution specifically on the missing wedge, we ran the alignment without this randomization step: that is, with the missing wedge always in the same orientation, as it exists in the original particles. Compared with the WBP, the deconvolution-processed average
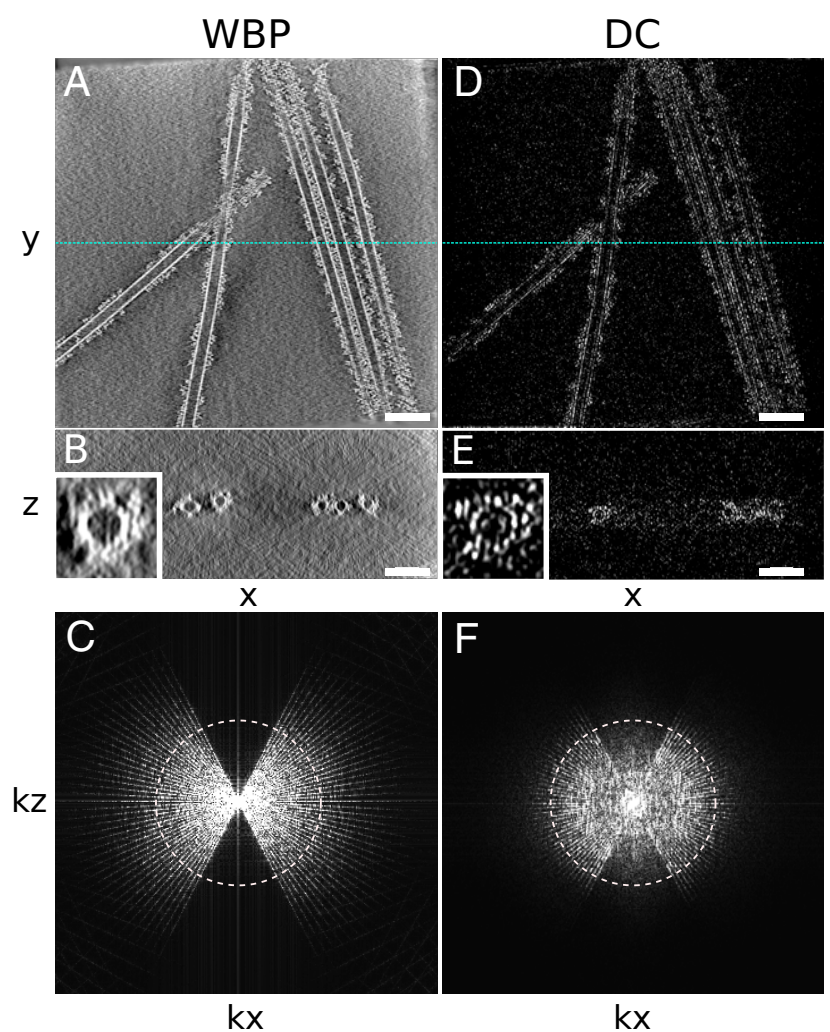

Fig. 6. Deconvolution of a tomogram of reconstituted microtubules. $(A) X Y$ slice of the WBP-reconstructed tomogram containing microtubules. $(B) \mathrm{XZ}$ view of the tomogram in $A$; the blue line in $A$ corresponds to the slice shown. Inset shows a larger view of one of the microtubules. (C) kxkz view showing the missing wedge. Inset shows a larger view of one of the microtubules. $(D)$ $X Y$ of the deconvolved (DC) tomogram. $(E) X Z$ of deconvolved tomogram in $D$; the blue line in $D$ corresponds to the slice shown. $(F)$ kxkz of the deconvolved tomogram. Dashed circles indicate $0.06-\AA^{-1}$ resolution. (Scale bars: $50 \mathrm{~nm}$.)

shows increased distinction between protofilaments in the direction of the missing wedge (Fig. 7D). Lastly, we used the alignment parameters generated from the azimuthally zeroed deconvolved subtomograms to the WBP particles to generate a WBP average

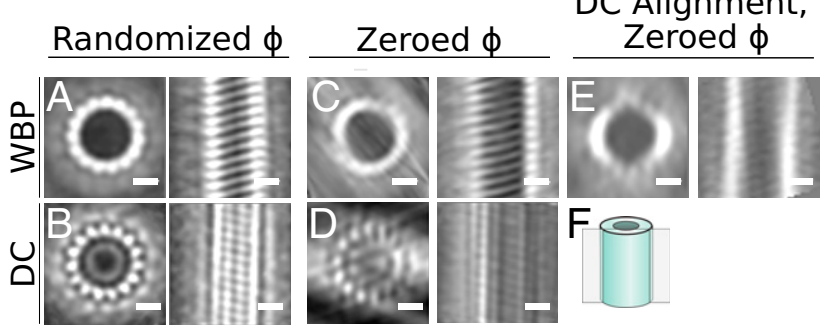

Fig. 7. Subtomogram analysis of WBP and a deconvolution-processed microtubule. In all panels, top and side views of the average are shown for the microtubule average obtained under the specified conditions. (A) Average from the WBP tomogram using a randomized azimuth angle $(\phi)$ averaging approach to compensate for the missing wedge (38). $(B)$ Average from the deconvolved (DC) tomogram using the same randomized $\phi$-scheme. (C) Average from the WBP tomogram, with initial constant $\phi$-angles for all particles, allowing the missing wedge to affect the average. (D) Average from the deconvolved tomogram using the same constant $\phi$ scheme. $(E)$ Average generated by applying the alignment parameters from the deconvolution uniform starting azimuth/restricted rotation alignment to the WBP particles. $(F)$ Schematic showing the location of the slice in the right-hand side image in each panel. (Scale bars: $10 \mathrm{~nm}$.) 
(Fig. $7 E$ ). Here, the deconvolution-aligned WBP average still shows a prominent missing wedge, similar to the WBP average generated by aligning WBP particles. This indicates that the improvement in the average from the deconvolved particles is not simply due to improved alignment but that the filling of the missing wedge is reducing distortions in $\mathrm{XZ}$, thereby improving the resulting subtomogram averages.

In addition to the experimental data explored in this paper, we used simulated tomography data to investigate the effect of deconvolution on a system with a known solution. While the noise models in simulated data are not fully comparable with experimental data, we reasoned that we should apply deconvolution to these data with the expectation that it recovers the initial structure accurately by filling in the missing information in the missing wedge area. We picked a X-ray crystallography structure of a microtubule from the Protein Data Bank (PDB; ID code $3 \mathrm{~J} 2 \mathrm{U})$ to generate a simulated cryo-EM map at $3.3-\AA$ r resolution using EMAN2 (60). Then, we simulated a tilt series of the density map (SI Appendix, Fig. S6), which was reconstructed using our standard WBP (SI Appendix, Fig. S7B). Deconvolution was applied to this simulated tomogram, resulting in a near-complete refilling of the missing wedge (SI Appendix, Fig. S7 D and $G$ ) and demonstrating that deconvolution works in idealized cases.

\section{Discussion}

We have successfully applied ER-DC to cryo-electron tomograms and demonstrated enhanced contrast compared with the WBP reconstructions, as well as reduced resolution anisotropy along the $z$ axis. In real space, one can follow membranes in the XZ plane of the deconvolved volume that were hardly visible in the WBP. In Fourier space, it is clear that portions of the missing wedge are filled in, and the distribution of voxel intensities changes significantly as a result of deconvolution. However, there are still several considerations for TEM deconvolution, and these are further discussed in SI Appendix.

First, the reality is that deconvolution acts as a filter for the data. The intensity of each voxel is modified in some fashion, and care must be taken in interpreting the deconvolved volume. Deconvolution has two parameters, for nonlinearity and smoothness, and the optimal values must be determined experimentally by systematically varying the parameters over several orders of magnitude; the parameter search quickly settles into basic convergent deconvolved images that look biologically reasonable (e.g., membrane bilayers are visible, ribosomes are distinct, etc.). At the end of the deconvolution process, one can usually settle on a few deconvolved images coming from a close smoothness parameter. These different deconvolved images are studied side by side comparing 3D volumes for details. The side by side images are very similar to one another, but subtle features between them exist. Crucial are the orthogonal Z-image planes for judging smoothness parameters and structure. There are a number of considerations for the deconvolution process, discussed in SI Appendix.

How does one know if the deconvolved structure is credible? In addition to the side by side study of several smoothness deconvolved images, a control raw WBP image must be studied alongside the deconvolved images, at several intensity scalings of the WBP data. Any feature uncovered in the deconvolved data would be searched for in the raw WBP data control and would have to be present in the WBP control. However, in our experience, the deconvolution process has never been observed to invent a structure that is not present in the WBP raw data control (29).

This study makes the statement that the missing wedge of information is substantially filled by deconvolution. Visually and in Fourier space representation, this is the case; however, this statement needs caution. We do not know if deconvolution will improve $\mathrm{Z}$ resolution for certain kinds of data, intensities, or different structures (e.g., of various sizes). It is possible that spaced periodic structures positioned on top of one another along $\mathrm{Z}$ in a tomogram are not resolved correctly in the deconvolved data.

A second point in the deconvolution discussion centers on what mathematics allows unobserved data to propagate from areas of observed data into their correct structural space. Since all image information can be decomposed into 3D Fourier representation, one is, in essence, saying that there is information in one region of Fourier space that can be extrapolated correctly into other regions of Fourier space by the deconvolution process. There are two examples from the inverse problems literature to reassure that such extrapolated information can indeed be real. The first one is called analytical continuation (AC) (ref. 61 and references therein), which is known in optics literature. The AC conjecture, taken from ref. 61, states that all image information can be decomposed into a Fourier transform, and a spatially bounded region of Fourier space can be expressed as an analytical function. The analytical function can be exactly known for a small region, and if there is no noise, the entire analytical function can be determined/extrapolated by AC. The extension can continue indefinitely, and this is a hallmark of AC (61). Noise is critical, and the analytical values become small as iterations progress as the function gets extended to higher-resolution regions of Fourier space, reasons that AC is little used (but see ref. 61). In the case of ER-DC, noise is heavily suppressed, and resolution extensions required are modest, suggesting that $\mathrm{AC}$ might work.

The second one is called compressive sensing reconstruction, used in modalities such as MRI (62) and tomography (63). It involves high-quality reconstruction from highly undersampled Fourier data and tomographic projections with a limited set of angles with regularization constructed using derivatives. Because of the way the derivative operator is related to the measurement operator (tomographic projection or Fourier transformation), high-quality reconstruction becomes possible from sparse Fourier samples or from tomographic projections from a limited set of angles. Although these theories are not directly extensible to our recovery problem, they reassure that extensions in Fourier space are possible, and hence, the filled-in missing wedges may be trusted if the resultant structures in the real space appear plausible.

Another independent argument supporting why the missing wedges could correctly be filled can be given from a statistical viewpoint. Recall that the regularization used in the ER-DC enforces certain hypothesized joint distribution of intensity and second-order derivative magnitude. It turns out that the backprojected images deviate significantly from this joint distribution. Hence, the minimization involved in ER-DC brings in a proper filling on the wedges such that 1) the resulting realspace image is consistent with the measured projections and 2) the resulting real-space image better matches with hypothesized distribution.

Third, deconvolution could have an impact on the electron dose required to obtain a suitable tomogram. The deconvolution process might allow other dose reduction steps, such as fewer tilts and lower beam intensity. In addition, there are several aspects of the deconvolution process that can be improved and are described in SI Appendix.

The deconvolution process filling in the missing wedges in Fourier space allows biological structures to be followed in three dimensions. This resolution is adequate to see: for example, gaps between the 10 -nm nucleosomes allowing a chromosome path to be followed. One imagines a two-step process for cellular tomography. First, the path of a structure is followed with the architecture discerned, a process greatly improved by deconvolution. Subsequently, after an architecture is determined, molecular features can be superimposed using averaging methods and molecular modeling (38). 


\section{Materials and Methods}

Sample Preparation. Yeast S. cerevisiae W303a cells were grown at $30{ }^{\circ} \mathrm{C}$ in YPD media (1\% yeast extract, $2 \%$ bactopeptone, and $2 \%$ glucose) to midlog phase, after which $5 \mu \mathrm{L}$ were deposited in a glow-discharged Quantifoil grid (200-mesh copper R2/1; Electron Microscopy Sciences), followed by manual blotting and plunge freezing in a 50/50 ethane propane mix (Airgas) using a custom-built manual plunger (Max Planck Institute of Biochemistry). HEK293T cells transfected with LRRK2-I2020T cells were prepared as described in ref. 38. In vitro reconstituted LRRK2-I2020T was prepared as described in ref. 56. For both yeast and HEK cells, frozen cells were micromachined on a Scios or an Aquilos 2 DualBeam FIB/scanning electron microscope (TFS). FIB milling was done as described in ref. 6.

Cryo-ET. Tilt series were obtained on a 300-kV Tecnai G2 Polara (TFS) or Titan Krios with a field emission gun, a GIF Quantum LS energy filter (Gatan), and a K2 Summit $4 \mathrm{k} \times 4 \mathrm{k}$-pixel direct electron detector (Gatan). Tilt series were acquired between $\pm 50^{\circ}$ and $\pm 70^{\circ}$, with increments of $2^{\circ}$ and $3^{\circ}$, tota electron doses between 70 and $100 \mathrm{e}^{-} / \AA^{2}$ at a target defocus of $5 \mu \mathrm{m}$, and a pixel size of 2.2 or $3.5 \AA$ using the SerialEM software (64) in low-dose mode. Bidirectional or dose-symmetric tomography acquisition schemes were used (16), corrected for the pretilt of the lamella where appropriate. Images acquired on the $\mathrm{K} 2$ detector were taken in counting mode, divided into frames of 0.075 to $0.1 \mathrm{~s}$

Tomogram Reconstruction. Tilt series were aligned and dose weighted by cumulative dose with MotionCorr2 (65). Dose-weighted tilt series were aligned and reconstructed using Etomo, part of the IMOD package (66) Patch tracking was used to define the model for fine alignment. The aligned tilt series were reconstructed using WBP to generate the 3D tomograms.

Deconvolution. A set of synthetic projections was generated with $x$ and $y$ dimensions and pixel spacing matching the tilt series that was used to make the original reconstructed volume. Each projection had a centered point source that is then convolved with the inverse Fourier transform of the CTF, generated using the defocus and astigmatism parameters estimated by CTFFIND4 (20). The convolved point source/CTF is then reconstructed using the same WBP used to generate the target reconstructed volume. Finally, the reconstruction is cropped to the same dimensions as the volume to be deconvolved, the 3D FFT of which will be used as the final PSF. Deconvolution is then run for 100 cycles using the generated PSF. A detailed description of the deconvolution procedure can be found in SI Appendix.

Subtomogram Analysis. Microtubule filaments were traced in Dynamo (59) to define coordinates and orientation. Single particles were defined every $4 \mathrm{~nm}$ along the filament, and subtomograms with a side length of $66 \mathrm{~nm}$ were then extracted from both the back-projected and the deconvolved tomograms using these coordinates. For both sets of particles, subtomograms were iteratively aligned over three rounds of two iterations each. The particles were aligned using a spherical alignment mask to minimize bias. For the first round, the alignment was constrained to a $180^{\circ}$ cone aperture, with no flip allowed and $20^{\circ}$ of azimuthal rotation, corresponding to the third Euler angle. Rounds 2 and 3 used $30^{\circ}$ and $10^{\circ}$ cone apertures, respectively, and azimuthal search ranges of $10^{\circ}$ and $2^{\circ}$, respectively. No symmetry was assumed in the alignment. Further details are in ref. 38 . To assess any compensation for the missing wedge, alignment was performed on particles with initial tables describing the particles orientation from 1) a blank table to set all particle orientations to zero and 2) a random table assigning each particle a random orientation.

To calculate averages for the autophagosome crystal subunit in the WBP and deconvolution tomograms, the first 50 particles were identified

1. E. Villa, M. Schaffer, J. M. Plitzko, W. Baumeister, Opening windows into the cell: Focused-ion-beam milling for cryo-electron tomography. Curr. Opin. Struct. Biol. 23, 771-777 (2013)

2. M. Marko, C. Hsieh, R. Schalek, J. Frank, C. Mannella, Focused-ion-beam thinning of frozen-hydrated biological specimens for cryo-electron microscopy. Nat. Methods 4 , 215-217 (2007).

3. A. Rigort et al., Focused ion beam micromachining of eukaryotic cells for cryoelectron tomography. Proc. Natl. Acad. Sci. U.S.A. 109, 4449-4454 (2012).

4. J. Harapin et al., Structural analysis of multicellular organisms with cryo-electron tomography. Nat. Methods 12, 634-636 (2015).

5. M. Schaffer et al., A cryo-FIB lift-out technique enables molecular-resolution cryo-ET within native Caenorhabditis elegans tissue. Nat. Methods 16, 757-762 (2019)

6. F. R. Wagner et al., Preparing samples from whole cells using focused-ion-beam milling for cryo-electron tomography. Nat. Protoc. 15, 2041-2070 (2020).

7. Y. W. Chang, C. L. Shaffer, L. A. Rettberg, D. Ghosal, G. J. Jensen, In vivo structures of the Helicobacter pylori cag type iv secretion system. Cell Rep. 23, 673-681 (2018).

8. V. Lucić, A. Leis, W. Baumeister, Cryo-electron tomography of cells: Connecting structure and function. Histochem. Cell Biol. 130, 185-196 (2008). manually in the deconvolved volume to generate an initial average. This initial average was used as a template for Dynamo's template-matching functionality and used to search for similar particles. A cross-correlation threshold of 0.38 was selected, below which many particles appeared as false positives by visual inspection. Using the coordinates and putative orientations from template matching, 82 particles were cropped from both the back-projected and deconvolved volumes. A global alignment was used on each dataset in two (even and odd sets) using the Dynamo subtomogram alignment function (59). Each half dataset was independently averaged and the dFSC was calculated between the resulting half averages (58). The alignment angles from the deconvolved particles were then applied to the WBP particles to create the average shown in Fig. $5 \mathrm{~A}$ and to the relative resolution by dFSC.

Simulated Data. To validate the effect of deconvolution on the missing wedge under ideal circumstances, a microtubule tomogram was generated from an existing crystal structure (PDB ID code 3J2U). After removing the chains that did not correspond to tubulin subunits forming the microtubule from the model in University of California, San Francisco (UCSF) Chimera (67), the PDB map was converted to a density map using the EMAN2 functionality e2pdb2mrc.py to convert it to a simulated density map, followed by the e2spt_simulation.py function to simulate a tilt series (60). At this stage, the simulated tilt series was an idealized example, with no CTF applied. e2spt_simulation.py defaults to simulating the particle as if it was embedded in $400 \mathrm{~nm}$ of vitreous ice, and the tilt series was binned to make the pixel size $3.3 \AA$ Apx to approximate the sampling often used in cellular tomograms. The CTF was simulated and applied to the synthetic tilt series with Priism's pfocusramp. The CTF parameters included a defocus of $-3.00 \mu \mathrm{m}$, with no astigmatism. A corresponding PSF was generated by applying the same CTF to a simulated point source tomogram derived from the simulated microtubule tilt series. The simulated tilt series was reconstructed by WBP and then deconvolved with the corresponding PSF

Data Availability. The tomograms and their corresponding deconvolutions have been deposited in the Electron Microscopy Database (yeast WBP and deconvolution data: accession nos. EMD-24433 and EMD-24434, respectively the tilt series and the corresponding tilt and defocus files: accession no. EMPIAR-10762; the inclusion body WBP and deconvolution data: accession nos. EMD-24435 and EMD-24436, respectively; and the tilt series and the corresponding tilt and defocus files: accession no. EMPIAR-10761). All the wrapper scripts necessary to perform the steps described are available at GitHub (https://github.com/Villa-Lab/ER-DC). ER-DC software and documentation can be obtained from D.A. and J.S. at UCSF. All other data are included in the manuscript and/or supporting information.

ACKNOWLEDGMENTS. We are indebted to Eric Branlund, whose computational efforts made this work possible. We thank Drs. Yifan Cheng (UCSF) David DeRosier (Brandeis and La Jolla), Robert Stroud (UCSF), Susan Taylor (University of California San Diego), and members of the laboratory of E.V. for discussions and support. We used samples and tomograms provided by Robert Buschauer, Reika Watanabe, Colin Deniston, and Andres Leschziner. We acknowledge the use of the University of California San Diego cryo-EM facility, which was built and equipped with funds from the University of California San Diego and an initial gift from Agouron Institute, and of the San Diego Nanotechnology Infrastructure of the University of California San Diego, a member of the National Nanotechnology Coordinated Infrastruc ture, supported by NSF Grant ECCS-1542148. This work was supported by NIH Grant R35GM118099 (to D.A.), NIH New Innovator Award DP2 GM123494 (to E.V.), NSF MRI Grant DBI 1920374 (to E.V.), and private funds from J.S M.E. is the Sam and Ayala Zacks Professorial Chair and head of the Irving and Cherna Moskowitz Center for Nano and Bio-Nano Imaging. E.V. is an investigator of the Howard Hughes Medical Institute.

9. Z. Ke et al., Promotion of virus assembly and organization by the measles virus matrix protein. Nat. Commun. 9, 1736 (2018)

10. J. Frank "Introduction: Principles of electron tomography" in Electron Tomography, J. Frank, Ed. (Springer, New York, NY, 2006), pp. 1-15.

11. J. A. Briggs, Structural biology in situ-the potential of subtomogram averaging Curr. Opin. Struct. Biol. 23, 261-267 (2013).

12. P. Zhang, Advances in cryo-electron tomography and subtomogram averaging and classification. Curr. Opin. Struct. Biol. 58, 249-258 (2019).

13. J. Böhning, T. A. M. Bharat, Towards high-throughput in situ structural biology using electron cryotomography. Prog. Biophys. Mol. Biol. 160, 97-103 (2021).

14. L. A. Baker, J. L. Rubinstein, Radiation damage in electron cryomicroscopy. Methods Enzymol. 481, 371-388 (2010).

15. T. Grant, N. Grigorieff, Measuring the optimal exposure for single particle cryo-EM using a $2.6 \AA \AA$ reconstruction of rotavirus VP6. eLife 4, e06980 (2015).

16. W. J. H. Hagen, W. Wan, J. A. G. Briggs, Implementation of a cryo-electron tomography tilt-scheme optimized for high resolution subtomogram averaging. J. Struct. Biol. 197, 191-198 (2017). 
17. H. P. Erickson, A. Klug, The Fourier transform of an electron micrograph: Effects of defocussing and aberrations, and implications for the use of underfocus contrast enhancement. Ber. Bunsenges. Phys. Chem 74, 1129-1137 (1970).

18. H. P. Erickson, A. Klug, Measurement and compensation of defocusing and aberrations by Fourier processing of electron micrographs. Philos. Trans. R. Soc. Lond. B Biol. Sci. 261, 105-118 (1971).

19. R. H. Wade, A brief look at imaging and contrast transfer. Ultramicroscopy 46,145 156 (1992).

20. A. Rohou, N. Grigorieff, CTFFIND4: Fast and accurate defocus estimation from electron micrographs. J. Struct. Biol. 192, 216-221 (2015).

21. M. Turk, W. Baumeister, The promise and the challenges of cryo-electron tomography. FEBS Lett. 594, 3243-3261 (2020).

22. A. S. Frangakis, R. Hegerl, Noise reduction in electron tomographic reconstructions using nonlinear anisotropic diffusion. J. Struct. Biol. 135, 239-250 (2001).

23. J. J. Fernández, S. Li, An improved algorithm for anisotropic nonlinear diffusion for denoising cryo-tomograms. J. Struct. Biol. 144, 152-161 (2003).

24. T. O. Buchholz, M. Jordan, G. Pigino, F. Jug, "Cryo-care: Content-aware image restoration for cryo-transmission electron microscopy data" in 2019 IEEE 16th International Symposium on Biomedical Imaging (ISBI 2019) (IEEE Computer Society, New York, NY, 2019), pp. 502-506.

25. X. Huang, S. Li, S. Gao, Exploring an optimal wavelet-based filter for cryo-ET imaging. Sci. Rep. 8, 2582 (2018).

26. D. Tegunov, P. Cramer, Real-time cryo-electron microscopy data preprocessing with Warp. Nat. Methods 16, 1146-1152 (2019).

27. R. P. Dougherty, Extensions of Damas and Benefits and Limitations of Deconvolution in Beamforming (AIAA International, 2005), vol. 3, pp. 2036-2048.

28. D. Sage et al., DeconvolutionLab2: An open-source software for deconvolution microscopy. Methods 115, 28-41 (2017).

29. B. Waugh et al., Three-dimensional deconvolution processing for STEM cryotomography. Proc. Natl. Acad. Sci. U.S.A. 117, 27374-27380 (2020).

30. T. Bepler, K. Kelley, A. J. Noble, B. Berger, Topaz-Denoise: General deep denoising models for cryoEM and cryoET. Nat. Commun. 11, 5208 (2020).

31. R. Yan, S. V. Venkatakrishnan, J. Liu, C. A. Bouman, W. Jiang, MBIR: A cryo-ET $3 \mathrm{D}$ reconstruction method that effectively minimizes missing wedge artifacts and restores missing information. J. Struct. Biol. 206, 183-192 (2019).

32. S. F. Gibson, F. Lanni, Experimental test of an analytical model of aberration in an oil-immersion objective lens used in three-dimensional light microscopy. J. Opt. Soc. Am. A 9, 154-166 (1992).

33. J. Swedlow, J. Sedat, D. Agard, "Deconvolution in optical microscopy" in Deconvolution of Images and Spectra, P. Jansson, Ed. (Academic Press, San Diego, CA, ed. 2, 1997), pp. 284-309.

34. Z. Kam, B. Hanser, M. G. Gustafsson, D. A. Agard, J. W. Sedat, Computational adaptive optics for live three-dimensional biological imaging. Proc. Natl. Acad. Sci. U.S.A. 98, 3790-3795 (2001).

35. P. Jansson, Ed., Deconvolution of Images and Spectra (Academic Press, San Diego CA, ed. 2, 1997).

36. M. Arigovindan et al., High-resolution restoration of 3D structures from widefield images with extreme low signal-to-noise-ratio. Proc. Natl. Acad. Sci. U.S.A. 110, 17344-17349 (2013)

37. S. Asano, B. D. Engel, W. Baumeister, In situ cryo-electron tomography: A postreductionist approach to structural biology. J. Mol. Biol. 428 (2 Pt A), 332-343 (2016).

38. R. Watanabe et al., The in situ structure of Parkinson's disease-linked LRRK2. Cell 182, 1508-1518.e16 (2020).

39. S. R. Carlsson, A. Simonsen, Membrane dynamics in autophagosome biogenesis. J. Cell Sci. 128, 193-205 (2015).

40. H. Chen, W. K. Clyborne, J. W. Sedat, D. A. Agard, "Priism: An integrated system for display and analysis of 3-d microscope images" in Proceedings of the SPIE/IS\&T 1992 Symposium on Electronic Imaging: Science and Technology, R. S. Acharya, C. J. Cogswell, D. B. Goldgof, Eds. (SPIE, Bellingham, WA, 1992), vol. 1660, pp. 784-790.

41. D. Castaño-Díez, G. Zanetti, In situ structure determination by subtomogram averaging. Curr. Opin. Struct. Biol. 58, 68-75 (2019).
42. J. Mahamid et al., Visualizing the molecular sociology at the HeLa cell nuclear periphery. Science 351, 969-972 (2016).

43. J. Hutchings, V. Stancheva, E. A. Miller, G. Zanetti, Subtomogram averaging of COPII assemblies reveals how coat organization dictates membrane shape. Nat. Commun. 9, 4154 (2018).

44. M. Khoshouei, S. Pfeffer, W. Baumeister, F. Förster, R. Danev, Subtomogram analysis using the Volta phase plate. J. Struct. Biol. 197, 94-101 (2017).

45. D. A. Grotjahn et al., Cryo-electron tomography reveals that dynactin recruits a team of dyneins for processive motility. Nat. Struct. Mol. Biol. 25, 203-207 (2018).

46. Z. Ke et al., Structures and distributions of SARS-CoV-2 spike proteins on intact virions. Nature 588, 498-502 (2020).

47. M. Obr, F. K. M. Schur, Structural analysis of pleomorphic and asymmetric viruses using cryo-electron tomography and subtomogram averaging. Adv. Virus Res. 105, 117-159 (2019)

48. C. Jiménez-Ortigosa et al., Preliminary structural elucidation of $\beta$-(1,3)-glucan synthase from candida glabrata using cryo-electron tomography. J. Fungi (Basel) 7, 1-13 (2021)

49. J. L. Ferreira et al., $\gamma$-proteobacteria eject their polar flagella under nutrient depletion, retaining flagellar motor relic structures. PLoS Biol. 17, e3000165 (2019).

50. B. Turoňová et al., In situ structural analysis of SARS-CoV-2 spike reveals flexibility mediated by three hinges. Science 370, 203-208 (2020).

51. J. Lin, T. Heuser, B. I. Carbajal-González, K. Song, D. Nicastro, The structural heterogeneity of radial spokes in cilia and flagella is conserved. Cytoskeleton (Hoboken) 69, 88-100 (2012).

52. Y. Fukuda, F. Beck, J. M. Plitzko, W. Baumeister, In situ structural studies of tripeptidy peptidase II (TPPII) reveal spatial association with proteasomes. Proc. Natl. Acad. Sci. U.S.A. 114, 4412-4417 (2017)

53. S. Asano et al., Proteasomes. A molecular census of $26 \mathrm{~S}$ proteasomes in intact neurons. Science 347, 439-442 (2015)

54. G. L. Weiss, A. K. Kieninger, I. Maldener, K. Forchhammer, M. Pilhofer, Structure and function of a bacterial gap junction analog. Cell 178, 374-384.e15 (2019).

55. B. A. Himes, P. Zhang, emClarity: Software for high-resolution cryo-electron tomography and subtomogram averaging. Nat. Methods 15, 955-961 (2018).

56. C. K. Deniston et al., Structure of LRRK2 in Parkinson's disease and model for microtubule interaction. Nature 588, 344-349 (2020).

57. H. Y. Liao, J. Frank, Definition and estimation of resolution in single-particle reconstructions. Structure 18, 768-775 (2010).

58. S. Dang et al., Cryo-EM structures of the TMEM16A calcium-activated chloride channel. Nature 552, 426-429 (2017).

59. D. Castaño-Díez, M. Kudryashev, M. Arheit, H. Stahlberg, Dynamo: A flexible, userfriendly development tool for subtomogram averaging of cryo-EM data in highperformance computing environments. J. Struct. Biol. 178, 139-151 (2012).

60. G. Tang et al., EMAN2: An extensible image processing suite for electron microscopy. J. Struct. Biol. 157, 38-46 (2007).

61. J. W. Goodman, Introduction to Fourier Optics (McGraw-Hill Companies, New York NY, ed. 2, 1968), pp. 161-165.

62. J. C. Ye, Compressed sensing MRI: A review from signal processing perspective. BMC Biomed. Eng. 1, 8 (2019).

63. R. Leary, Z. Saghi, P. A. Midgley, D. J. Holland, Compressed sensing electron tomography. Ultramicroscopy 131, 70-91 (2013).

64. M. Schorb, I. Haberbosch, W. J. H. Hagen, Y. Schwab, D. N. Mastronarde, Software tools for automated transmission electron microscopy. Nat. Methods 16, 471-477 (2019).

65. S. Q. Zheng et al., MotionCor2: Anisotropic correction of beam-induced motion for improved cryo-electron microscopy. Nat. Methods 14, 331-332 (2017).

66. J. R. Kremer, D. N. Mastronarde, J. R. McIntosh, Computer visualization of threedimensional image data using IMOD. J. Struct. Biol. 116, 71-76 (1996).

67. E. F. Pettersen et al., UCSF Chimera-A visualization system for exploratory research and analysis. J. Comput. Chem. 25, 1605-1612 (2004). 